\title{
Understanding the Composition of Dispersed Phases in Silicon-Rich Metal-Silicon Alloys by Parsing SEM/EDS Hyperspectral Images
}

\author{
Jeremy M. Beebe ${ }^{1}$, Matthew A. Gave ${ }^{1}$, Joseph R. Sootsman ${ }^{1}$, Alitha A. Klele ${ }^{1}$, James R. Young ${ }^{1}$ and \\ Vasgen A. Shamamian ${ }^{1}$ \\ 1. The Dow Chemical Company, Midland, MI, USA.
}

Silicon is a brittle material and possesses no intrinsic mechanism for arresting crack propagation. It is possible to improve the fracture toughness of silicon by constructing silicon-rich metal-silicon alloys which contain dispersed silicide phases that arrest or deflect propagating cracks [1]. There exist an array of simple $\mathrm{M} / \mathrm{Si}(\mathrm{M}=$ metal) binary systems where the metals added to the melt result in spontaneous formation of a reinforcing disilicide eutectic $\left(\mathrm{MSi}_{2}\right)$ phase during solidification. Additional toughening beyond what is possible in these binary systems can be imparted by adding additional metals to the melt to cause a more torturous microstructure which results in better crack deflection.

While studying metal/silicon combinations to optimize alloy fracture toughness, we discovered that ternary alloys that employ metals that do not form a solid solution in each other always result in physically separate phases of $\mathrm{ASi}_{2}$ and $\mathrm{BSi}_{2}$, where $\mathrm{A}$ and $\mathrm{B}$ denote the two metals used. In these separate phases, SEM/EDS analysis indicates slight (2-3\%) solubility of A in B and B in A, with no concentration variation throughout the microstructure. That is, if $3 \% \mathrm{~A}$ is found in one $\mathrm{BSi}_{2}$ inclusion, $3 \% \mathrm{~A}$ will be found in all $\mathrm{BSi}_{2}$ inclusions (Fig. 1).

When the metals used to form the dispersed silicide phases form a solid solution, one might expect to observe phases of $\mathrm{A}_{\mathrm{x}} \mathrm{B}_{\mathrm{x}-1} \mathrm{Si}_{2}$, where $\mathrm{X}$ is a constant defined by the relative quantities of $\mathrm{A}$ and $\mathrm{B}$ added to the melt. Indeed, there is literature precedent for the formation of solid solutions of $\mathrm{CrSi}_{2}$ and $\mathrm{VSi}_{2}$ $[2,3]$. However, that is not what is observed in these materials. Instead, each individual disilicide inclusion is found to have a composition of $A_{x} B_{x-1} S_{2}$, where $X$ varies continuously across the inclusion. This is observed in backscattered electron images as a grayscale gradient, and manifests in EDS element maps as a color gradient (Fig. 2).

To better understand how changes in input metal concentration impact the silicide composition distribution, element concentration histograms were constructed by interrogating EDS hyperspectral images. To accomplish this, high-quality EDS intensity maps were collected using a Bruker XFlash 5030 SDD. At a map resolution of 256 x 192 pixels and an X-ray count rate of approximately $25 \mathrm{kcps,}$ an acquisition time of 80 minutes per map was required to reach an acceptable signal-to-noise ratio. Once a high-quality image was obtained, the QMap function of the Bruker Esprit software was used to convert the intensity maps to element concentration maps. These maps were exported as text files and manipulated in Igor Pro. X-Y locations corresponding to dispersed phases were identified using the expected concentration of $\mathrm{Si}$ in a disilicide coupled with an uncertainty budget. All locations with a $\mathrm{Si}$ concentration outside the specified range were eliminated from consideration, because these would correspond to 1) the continuous $\mathrm{Si}$ phase or 2) the $\mathrm{Si}$ /silicide interface or 3) locations where Al was present (in quaternary alloys). After downselecting the concentration map data to include only locations that corresponded to disilicide inclusions, histograms of the concentrations of A and B were generated to better understand the average concentrations of $\mathrm{A}$ and $\mathrm{B}$ present in the inclusions. 
The concentration histograms generated by this process often show bimodal behavior, indicating that there are specific compositions that are favored (Fig. 3). Altering the relative concentration of A and B in the melt is found to shift the mode position in these histograms. There is no apparent precedent for this behavior in the literature, and it would not have been possible to identify it without EDS hyperspectral image interrogation.

\section{References:}

[1] D Fischer in "Development of in situ Toughened Silicon-Rich Alloys: A New Class of Castable Engineering Ceramics", (Massachusetts Institute of Technology, Cambridge) p. 26.

[2] "Handbook of Ternary Alloy Phase Diagrams" ed. P Villars, A. Prince, H. Okamoto, (ASM International, Materials Park).

[3] K Tanaka et al., Materials Science and Engineering A 261 (1999), p. 158

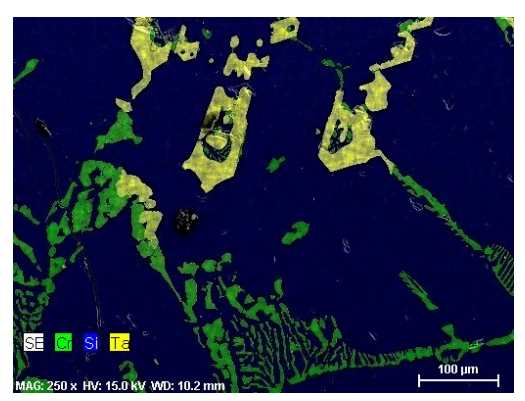

Figure 1. Microstructure of a $\mathrm{Cr} / \mathrm{Ta} / \mathrm{Si}$ alloy. The green phase is $\mathrm{CrSi}_{2}$, the yellow phase is $\mathrm{TaSi}_{2}$, and the blue phase is $\mathrm{Si}$. Cr and Ta do not form a solid solution, and form separate disilicide phases.

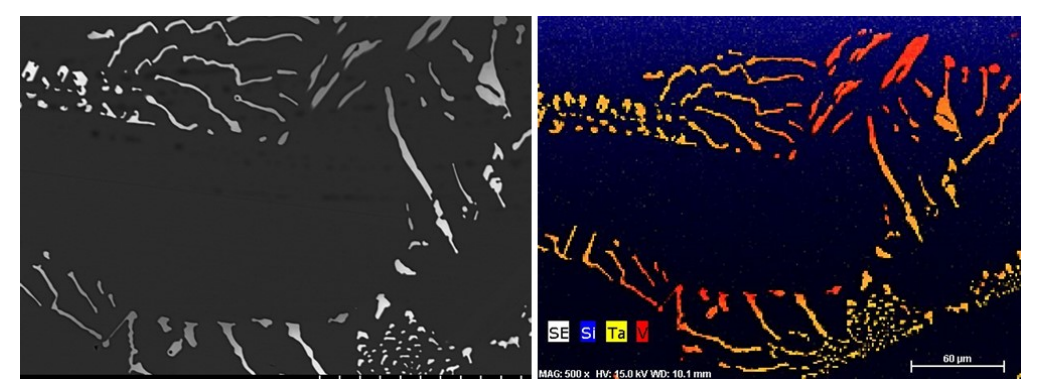

Figure 2. Microstructure of a Ta/V/Si alloy. The disilicide phases show contrast gradients in the backscattered electron image and color gradients in the EDS map consistent with a varying composition.
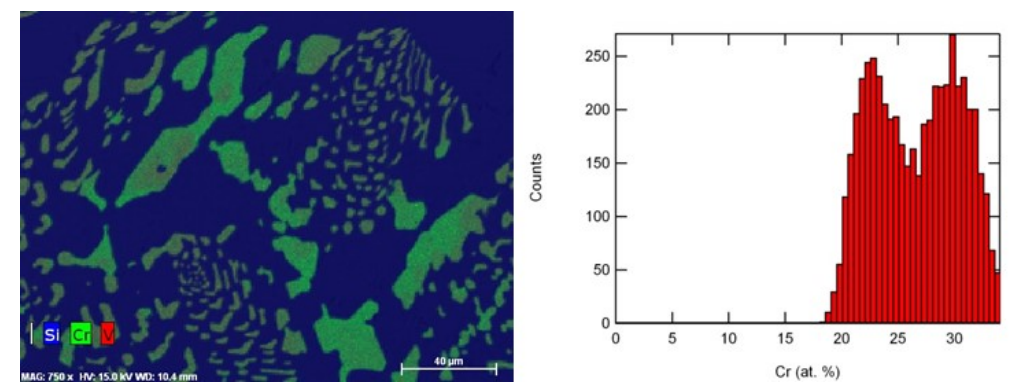

Figure 3. Microstructure of a $\mathrm{Cr} / \mathrm{V} / \mathrm{Si}$ alloy. The histogram shows the $\mathrm{Cr}$ distribution present in the dispersed disilicide phases. 